\title{
Réformer le mandat du bibliothécaire scolaire : une question de survie
}

\author{
Changing the Role of the School Librarian: A Question of
} Survival

\section{Renovar la autoridad del bibliotecario escolar: una cuestión de supervivencia}

\section{Brigitte Moreau}

Volume 57, numéro 2, avril-juin 2011

Les services d'information pour les jeunes : actualités et perspectives

URI : https://id.erudit.org/iderudit/1028877ar

DOI : https://doi.org/10.7202/1028877ar

Aller au sommaire du numéro

\section{Éditeur(s)}

Association pour l'avancement des sciences et des techniques de la documentation (ASTED)

\section{ISSN}

0315-2340 (imprimé)

2291-8949 (numérique)

Découvrir la revue

Citer cet article

Moreau, B. (2011). Réformer le mandat du bibliothécaire scolaire : une question de survie. Documentation et bibliothèques, 57(2), 121-125. https://doi.org/10.7202/1028877ar
Résumé de l'article

Cet article propose une vision du mandat et du rôle d'un bibliothécaire professionnel dans une commission scolaire qui collent aux exigences pédagogiques de l'école et répondent aux besoins d'une éducation évolutive en phase avec le développement social actuel. Il cherche notamment à rehausser l'image du bibliothécaire scolaire en démontrant comment celui-ci peut accroître sa crédibilité en adoptant une posture professionnelle. En outre, il met l'accent sur la nécessité du travail en intermédiation avec les différents acteurs du monde de l'éducation entre eux et, plus largement, en lien avec la communauté, afin de maximiser la cohérence des apprentissages offerts aux élèves. Enfin, il expose brièvement les actions de concertation à l'œuvre dans le monde de la bibliothéconomie scolaire au Québec.
Tous droits réservés (C) Association pour l'avancement des sciences et des techniques de la documentation (ASTED), 2011
Ce document est protégé par la loi sur le droit d'auteur. L'utilisation des services d'Érudit (y compris la reproduction) est assujettie à sa politique d'utilisation que vous pouvez consulter en ligne.

https://apropos.erudit.org/fr/usagers/politique-dutilisation/ 


\title{
Réformer le mandat du bibliothécaire scolaire : une question de survie
}

\author{
BRIGITTE MOREAU \\ Bibliothécaire, Commission scolaire de la Pointe-de-l'Île \\ brigitte-moreau-2@cspi.qc.ca
}

\section{RÉSUMÉ | ABSTRACTS | RESUMEN}

Cet article propose une vision $d u$ mandat et $d u$ rôle d'un bibliothécaire professionnel dans une commission scolaire qui collent aux exigences pédagogiques de l'école et répondent aux besoins d'une éducation évolutive en phase avec le développement social actuel. Il cherche notamment à rehausser l'image du bibliothécaire scolaire en démontrant comment celui-ci peut accroittre sa crédibilité en adoptant une posture professionnelle. En outre, il met l'accent sur la nécessité du travail en intermédiation avec les différents acteurs du monde de l'éducation entre eux et, plus largement, en lien avec la communauté, afin de maximiser la cohérence des apprentissages offerts aux élèves. Enfin, il expose brièvement les actions de concertation à l'ceuvre dans le monde de la bibliothéconomie scolaire au Québec.

\section{Changing the Role of the School Librarian :} $A$ Question of Survival

This article outlines a new vision of the mandate and role of the professional librarian in a school board; this new role is consistent with the pedagogical requirements of the school and meets the changing educational needs of today's society. It also challenges the image of the school librarian to show how he or she may increase his or her credibility by adopting a more professional stance. It also highlights the importance of establishing working relationships with stakeholders involved in education within the school and in the community in order to maximise the coherence of learning by the students. It also briefly describes the efforts for more and better collaboration within the school library network of Québec.

\section{Renovar la autoridad del bibliotecario} escolar : una cuestión de supervivencia

Este artículo propone una visión de la autoridad y de la función de un bibliotecario profesional dentro de una comisión escolar, que se ciñen a las exigencias pedagógicas de la escuela y responden a las necesidades de una educación evolutiva conforme al desarrollo social actual. Busca especialmente realzar la imagen del bibliotecario escolar demostrando de qué manera puede acrecentar su credibilidad mediante una postura profesional. Además, destaca la necesidad del trabajo de intermediación entre los diferentes participantes del mundo de la educación y, especialmente, en relación con la comunidad, a fin de maximizar la coherencia de los aprendizajes ofrecidos a los alumnos. Por último, expone brevemente las acciones de concertación a la obra en el mundo de la bibliotecología escolar en Quebec.

\section{Introduction}

A RECONDUCTION du Plan d'action sur la lecture à l'école (PALE) du ministère de l'Éducation, du Loisir et du Sport (MELS) en 2008 comporte une mesure supplémentaire au programme qui prévoit l'embauche de bibliothécaires professionnels dans le réseau des commissions scolaires du Québec sur une période de 10 ans; cette mesure est également réitérée dans le Plan d'action pour l'amélioration du français (PAF) (2008), du même ministère.

«Le ministère de l'Éducation, du Loisir et du Sportpoursuivra le réinvestissement qui a débuté en 2004-2005 dans les bibliothèques scolaires, en contribuant financièrement à l'acquisition de nouvelles ressources, et permettra aux commissions scolaires d'embaucher une vingtaine de bibliothécaires par année pendant dix ans, soit un total de 200 nouveaux bibliothécaires. "

(Québec. Ministère de l'Éducation, du Loisir et du Sport, 2008)

Nous sommes actuellement dans la troisième année de l'application de cette mesure et, déjà, une cinquantaine de nouveaux postes de bibliothécaire scolaire ont été créés ${ }^{1}$. Ce plan d'embauche dans le réseau des commissions scolaires québécois crée une impulsion sans précédent pour un réinvestissement majeur des professionnels dans le monde de l'enseignement primaire et secondaire, secteur laissé vacant depuis de trop nombreuses années. Les défis sont de taille et seules de nouvelles perspectives de travail seront garantes du succès d'une implantation durable et définitive des bibliothécaires professionnels dans ce réseau. L'avenir de notre profession, en milieu scolaire, dépend largement de notre écoute du milieu, de ses besoins et d'un ajustement pour comprendre et utiliser le langage pédagogique qui lui est propre. Il ne s'agit pas de recycler d'anciennes pratiques, l'heure est à un changement majeur de la perception de nos mandats et de notre rôle dans le système d'éducation.

Qu'a-t-il bien pu se passer entre les idéaux des années 1960 portés par le Rapport Parent (Rapport de la Commission royale d'enquête..., 1963-1964) et la triste

1. Information obtenue lors des journées de formation pour les nouveaux bibliothécaires scolaires, offertes par le MELS les 20 et 21 octobre 2010. 
réalité du terrain de la bibliothéconomie en milieu scolaire 50 ans plus tard? Avant le plan d'embauche, on ne comptait plus que quelques rares bibliothécaires professionnels disséminés ici et là et occupant des postes aux fonctions disparates (Charest et al., 2008 : 32). Après plusieurs années d'observations empiriques sur le terrain, nous émettons l'hypothèse que les facteurs de ce déséquilibre sont sûrement multiples et complexes, et probablement liés à une certaine intransigeance d'un discours bibliothéconomique pur et dur, sans concession pour le contexte scolaire, doublé d'un certain repli sur soi qui ont fait disparaitre, peu à peu, les bibliothécaires professionnels du réseau scolaire. Que l'hypothèse émise soit conforme ou non, ce constat d'échec est, quant à lui, bien réel. Et il constitue la plateforme à partir de laquelle nous devons maintenant reconstruire et consolider l'avenir.

Cet article ne sera pas le lieu d'une interprétation ou d'une analyse de ces tristes années du démantèlement du réseau des bibliothèques scolaires au Québec, mais à partir de ce constat, nous tenterons de baliser une nouvelle voie vers une implantation réussie de la bibliothéconomie en milieu scolaire.

\section{La mission de la bibliothèque et du bibliothécaire scolaires ${ }^{2}$}

Dans l'école, la bibliothèque constitue un milieu idéal pour les apprentissages, toutes disciplines confondues. Elle est un catalyseur de compétences et de connaissances; elle favorise la synergie d'un environnement humain, physique et virtuel, et l'énoncé de sa mission se lit comme suit :

\section{«La bibliothèque de l'école est un environne- ment éducatif et culturel qui contribue aux apprentissages et à l'enseignement, en adéqua- tion avec les objectifs de l'école, tout en favo- risant le développement personnel, culturel et social de l'élève3.»}

La bibliothèque est bien davantage qu'un lieu dédié à la consultation et aux emprunts de documents, elle est un service pédagogique essentiel, intégré au cœur de l'école, pour les enseignants et les élèves. En outre, elle représente, pour la majorité des jeunes d'âge scolaire, le premier lieu culturel à leur portée. Cependant, pour être à la hauteur d'une telle mission, l'intégrité du lieu doit être respectée. Ainsi, la bibliothèque ne doit ni servir de local de punition, ni être utilisée comme un dépôt de livres, ni servir à toutes autres fins que celles de ses activités propres.

2. Cette section s'inspire d'un document de travail interne rédigé par l'auteure sous le titre provisoire Définition du poste de bibliothécaire à la Commission scolaire de la Pointe-de-1'lle, juillet 2010; le document sera rendu public sur le site Web de la commission scolaire (<www.cspi.qc.ca/ $>$ ) après son approbation officielle.

3. Cette mission est inspirée de celle qui est énoncée dans le document de réflexion La bibliothèque scolaire, mission et objectifs, Montréal: APSDS, 1991, 11.
La mission de la bibliothèque est l'assise du mandat du bibliothécaire scolaire qui travaille à la promotion culturelle et à l'utilisation pédagogique du livre et des bibliothèques dans toutes les écoles du territoire de sa commission scolaire. Nous pourrions illustrer le rôle d'un bibliothécaire par les différentes actions qui le caractérisent: il planifie, structure, élabore, conseille, gère, supervise, organise, etc. Ses actions relèvent de la structuration d'un service plutôt que de la mise en application, laquelle pourrait, dans un monde idéal, être confiée aux techniciens en documentation.

En tant que professionnel dans une commission scolaire, son principal mandat est sa fonction conseil, qui se décline sur trois niveaux d'intervention : auprès des enseignants et des directions d'école, auprès des techniciens en documentation et auprès des conseillers pédagogiques, dans le but d'intégrer la bibliothèque à la vie pédagogique et culturelle de l'école.

À ce mandat, vient s'ajouter celui, indispensable, de son rôle en tant que passeur culturel, qui propose une solution de rechange pour entrer dans l'univers littéraire et documentaire. Son approche différenciée de celle des pédagogues par son rapport à la littérature, qui n'est jamais en lien direct avec une évaluation, lui confère un statut privilégié qui donne libre cours à une versatilité propice croyons-nous à l'émergence de nouvelles pratiques. Par exemple, une approche littéraire qui favorise la découverte plutôt que le travail didactique donne à l'élève une autre vision de la lecture, dans laquelle il peut s'investir personnellement. Ainsi, la recherche d'une combinaison entre l'enseignement de la lecture et une appropriation personnelle de l'acte de lire est une formule gagnante déjà appliquée par de plus en plus de pédagogues, et qui consiste à faire vivre une expérience littéraire. Plutôt que de l'utiliser simplement comme un support de l'enseignement, le livre devient le moteur d'une véritable expérience émotionnelle qui enrichit les apprentissages et aide les élèves à s'approprier les mécanismes cognitifs liés à la lecture. Plusieurs enseignants, surtout au primaire, mais aussi au secondaire, affectionnent ce genre de relation entre l'aspect didactique et l'expérience personnelle. Nous pensons qu'en soutenant ces démarches, le bibliothécaire, qui exerce d'abord un rôle de passeur, peut également devenir une ressource centrale pour soutenir ces pédagogues. Cependant, pour être viable, une telle approche demande que soit d'abord instauré un climat de complicité entre tous les acteurs de l'école, une connivence nécessaire entre le bibliothécaire et les conseillers pédagogiques, les enseignants, les membres de la direction et les techniciens en documentation. Or, ce lien de crédibilité n'est pas un acquis et demeure l'un des enjeux les plus délicats avec lequel les nouveaux bibliothécaires devront composer pour redéfinir leur rôle.

Enfin, le mandat du bibliothécaire s'élargit vers la communauté par la représentation et le développement de partenariats avec les instances sociales et les tables 
de concertation qui militent en faveur de la lecture chez les jeunes, les bibliothèques municipales et nationales, et tout autre organisme et institution culturels ou scientifiques. Par exemple, une implication dans les projets menés dans le cadre du Programme d'aide à l'éveil à la lecture et à l'écriture dans les milieux défavorisés (PAÉLÉ) du MELS4, qui encourage le travail en intermédiation avec différents acteurs communautaires et municipaux que sont: les organismes communautaires dont la mission est liée à la famille et à l'enfance, les bibliothèques publiques, les municipalités, les gens d'affaires, les écoles et les commissions scolaires, ainsi que l'implication de différents ministères 5 , sur des territoires préalablement identifiés "milieux défavorisés» par le gouvernement. Plusieurs initiatives du genre se sont développées au Québec. À titre d'exemple, le Comité ÉLÉ de Montréal-Nord, dont la maxime est "Grandira qui LIRA ", compte déjà de nombreuses initiatives partenariales. Le support soutenu de la Commission scolaire de la Pointe-de-l'île s'y concrétise en dégageant ses professionnels pour mettre en œuvre des activités qui visent à soutenir les parents dans l'initiation de leurs enfants d'âge préscolaire à la littératie, pour augmenter leurs chances de réussite dans le parcours scolaire.

\section{Rôles du bibliothécaire scolaire}

Dans les écoles, les interventions d'un bibliothécaire scolaire ont lieu à plusieurs niveaux, tant sur les plans humain et physique que pédagogique et culturel : dans la formation destinée aux enseignants, dans l'organisation structurelle des bibliothèques et dans une approche pédagogique et culturelle pour toutes les interventions. Ainsi, sur le plan humain, les bibliothécaires peuvent contribuer à améliorer l'offre de service des bibliothèques scolaires en soutenant des équipes de techniciens, un peu à l'image des conseillers pédagogiques auprès des enseignants, pour faire en sorte que ceux-ci accroissent leur crédibilité auprès des enseignants et prennent réellement leur place dans la vie pédagogique de l'école. Sur le plan physique, ce peut être une restructuration de l'offre de services de façon à maximiser l'utilisation des ressources afin que la bibliothèque devienne un service pédagogique et culturel essentiel et incontournable de l'école. À l'heure actuelle, les bibliothèques scolaires sont plus souvent perçues comme des dépôts de livres et bien peu sont véritablement intégrées à la vie pédagogique de leur école; "l'animation de la bibliothèque scolaire

\footnotetext{
4. Pour plus d'information sur ce programme, consulter <www.mels.gouv.qc.ca/

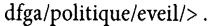

5. Sont impliqués dans ce projet le ministère de l'Éducation, du Loisir et du Sport (MELS), le ministère de la Culture, des Communications et de la Condition féminine (MCCCF), le ministère de la Santé et des Services sociaux (MSSS), le ministère de la Famille et des Aînés (MFA) et le ministère de l'Emploi et de la Solidarité sociale (MESS).
}

est le service le moins fréquent; il est offert par un peu plus du tiers des commissions scolaires (36,3\%). " (Charest et al., 2008 : 29). Dans les écoles primaires, ces actions seront menées en support au comité de bibliothèque; dans les écoles secondaires, elles seront proposées aux techniciens qui présenteront ces améliorations aux différentes tables pédagogiques de l'école. Par ailleurs, sur le plan pédagogique et culturel, des formations peuvent être élaborées par des bibliothécaires à l'intention des élèves et des enseignants; ces formations peuvent porter sur le développement des compétences informationnelles, ou encore sur la littérature jeunesse, avec présentation des différents genres, et proposant des pistes d'intégration pédagogiques en lien avec le programme, en collaboration avec les conseillers pédagogiques, ou encore des veilles théoriques s'adressant à ces derniers et aux enseignants. Finalement, l'idée maîtresse qui sous-tend ces actions, c'est qu'il importe de toujours considérer qu'elles sont faites pour le bénéfice des élèves. Sur le plan pédagogique et culturel, la contribution d'un bibliothécaire scolaire peut devenir véritablement enrichissante pour eux. Bien sûr, il sera toujours essentiel de se demander ce qu'un bibliothécaire peut bien changer à la vie d'un élève. Ce regard réflexif sur nos pratiques nous oblige à constamment repenser notre rôle en tant qu'éducateur et passeur culturel auprès des élèves, afin de conserver un dynamisme qui s'actualise aux besoins évolutifs des générations qui se succèdent dans nos écoles. En fait, un bibliothécaire concrétise des apprentissages conçus pour les élèves en leur procurant un lieu structuré pour ce faire, les bibliothèques, et en soutenant le développement de leurs compétences informationnelles, par le biais de formations proposées aux enseignants et le soutien aux équipes techniques. De plus, en tant que passeur culturel, le bibliothécaire peut contribuer à fortifier chez l'élève son projet de devenir lecteur ${ }^{6}$. Ainsi, le recours à une offre diversifiée de lecture de qualité et bien adaptée aux élèves peut faire une différence considérable sur la perception qu'auront d'euxmêmes ces jeunes lecteurs en formation. La littérature jeunesse et jeune adulte offre une quantité impressionnante de ressources, souvent peu exploitées parce que méconnues des pédagogues (qui sont bien occupés par ailleurs), qui pourront influencer favorablement la préhension de la lecture et le désir de devenir lecteur. En fait, notre rôle est justement de présenter ces multiples ressources aux pédagogues, en modélisant parfois quelques approches, afin de les soutenir dans leur tâche, parfois ingrate, de faire apprendre à lire. Notre rôle est plus amène, puisqu'il se situe davantage dans l'aspect ludique du bonheur de lire. Profitons-en!

\footnotetext{
6. Expression souvent employée dans les différents travaux de Christian Poslaniec, notamment.
} 


\section{Le travail en collégialité}

Tel un écosystème intégré, les mandats du bibliothécaire s'influencent mutuellement afin d'optimiser l'utilisation pédagogique et culturelle de la bibliothèque par tous les acteurs de l'école: enseignants, élèves, direction, professionnels et tout autre intervenant. Il s'agit de rendre la bibliothèque complémentaire à la salle de cours, d'en faire son prolongement quasi organique, son complément direct. Pour ce faire, le contexte virtuel de la bibliothèque doit être davantage exploité, ne serait-ce que par le recours quotidien au catalogue informatisé dans la classe, et ce, dès l'école primaire.

$\mathrm{Au}$ même titre que les conseillers pédagogiques, les bibliothécaires des commissions scolaires sont des professionnels qui ont une fonction de conseil. De fait, ils constituent une ressource pédagogique supplémentaire pour répondre aux besoins des enseignants. Ainsi, dans l'accompagnement de ces derniers, une collaboration étroite avec les conseillers pédagogiques doit être favorisée afin d'assurer la cohérence des interventions et des formations de chacun pour privilégier des pratiques complémentaires. En fait, le travail du bibliothécaire s'apparente à celui d'un conseiller pédagogique «transversal $»$, dans le sens où ses interventions touchent l'ensemble de la communauté apprenante et toutes les disciplines.

En plus des formations proposées aux enseignants pour intégrer la littérature et la bibliothèque dans leur enseignement, des mises en contexte par modélisation sont souhaitables. Ainsi, il ne s'agit pas uniquement de dégager les cadres théoriques de meilleures pratiques pédagogiques, mais aussi d'en démontrer la pertinence en classe. Ce type d'intervention donne au bibliothécaire un accès direct aux élèves, dans un contexte expérimental favorisant l'avancement de ses propres recherches.

Pour favoriser une utilisation maximale de la bibliothèque, le bibliothécaire doit s'assurer de l'adéquation entre le service qu'il prône en formation auprès des enseignants et les ressources disponibles dans chacune des écoles. Une synergie similaire à celle attendue avec les conseillers pédagogiques doit alors se manifester en regard des équipes de techniciens en documentation de la commission scolaire, pour ce qui concerne le soutien à l'organisation matérielle, informatique et intellectuelle de la bibliothèque. Ainsi, le bibliothécaire rédige les documents administratifs qui seront la clé de voûte de cet écosystème. Ces documents doivent être approuvés par la commission scolaire pour baliser une gestion concertée, contrôler les actions menées dans la bibliothèque et uniformiser l'ensemble des services offerts aux élèves, par exemple :

7. Selon l'expression empruntée à Marie-Christine Savoie, bibliothécaire à la Commission scolaire Des Chênes.
- une politique de développement de collections qui précise les modalités d'acquisition de documents et ressources numériques, incluant des outils encadrant la prise d'inventaire, l'élagage et l'évaluation de la collection ;

- un guide de la bibliothèque qui présente les rôles et fonctions de chacun des intervenants : enseignants, direction, élèves, bénévoles, techniciens en documentation, bibliothécaires et autres, en décrivant l'encadrement nécessaire à la réalisation d'un objectif commun, celui d'optimiser le service de la bibliothèque ;

- une évaluation des ressources numériques pour favoriser les abonnements collectifs.

La mise en place de rencontres statutaires dans le but d'offrir de la formation continue aux techniciens en documentation et de les sortir de leur isolement est une autre action qui porte fruit. Ainsi peuvent être instaurées trois ou quatre rencontres annuelles de formation et d'informations, qui deviennent un lieu d'échange sur les pratiques et une occasion de mise à jour des connaissances visant à accroître l'efficience des techniciens en documentation sur le terrain et, d'un même souffle, leur crédibilité auprès du personnel enseignant et des membres de la direction. De plus, ce genre de réunion consolide l'esprit d'équipe, raffermit le sentiment d'appartenance à la commission scolaire et à ses objectifs, optimise les ressources par le partage des pratiques respectives tout en uniformisant l'offre de service pour l'ensemble des élèves de la commission scolaire.

Concrètement, le rôle des bibliothécaires sera de répondre à des attentes très précises, dans cet esprit de collégialité, telles que: Comment utiliser le livre comme outil pédagogique? Comment donner le goût de lire? Comment développer les habiletés informationnelles des élèves? Comment dynamiser l'utilisation de la bibliothèque de l'école et en faire un lieu d'apprentissage interactif ? Comment intégrer les technologies de l'information et utiliser les réseaux virtuels et les ressources numériques pour étendre la portée des actions pédagogiques au quotidien et même à la maison?

En considérant le travail en amont effectué par une équipe de bibliothécaires dans une commission scolaire, la bibliothèque de l'école doit devenir un centre d'apprentissage interactif où tous les intervenants de l'école collaborent afin d'uniformiser les pratiques pédagogiques, en tout temps, tout en respectant l'intégrité du lieu qu'est la bibliothèque.

\section{En conclusion : le bibliothécaire en quête d'une nouvelle image}

Actuellement, les bibliothécaires scolaires sont en train de s'organiser en formant différents groupes de travail pour élaborer et construire l'avenir. Ainsi, un 
mécanisme de contact et d'information a été mis en place avec la création d'un "Google group", devenu l'instrument d'un réseau d'échange et de discussion des plus stimulants. C'est par ce réseau que s'est constitué un premier groupe de travail, le Comité de la norme, qui cherche à redéfinir la bibliothèque scolaire et la place qu'elle doit prendre dans le système d'éducation québécois à partir de critères structurants, qualitatifs et quantitatifs. En tout, 13 bibliothécaires provenant de dix commissions scolaires se réunissent régulièrement depuis juin 2010 pour mener cette tâche à terme. Le travail en cours nous amène bien sûr à poser un regard réflexif sur la nature du travail du bibliothécaire dans une commission scolaire et les enjeux d'une bibliothèque au $\mathrm{XXI}^{\mathrm{e}}$ siècle. Parallèlement aux travaux de ce groupe, des initiatives sur le terrain ont vu le jour et différents groupes de travail régionaux se sont constitués. Ainsi, une première Table régionale de bibliothécaires, celle de Montréal, qui regroupe 18 bibliothécaires des cinq commissions scolaires de l'Île ${ }^{8}$, a été officialisée à l'automne 2010 par la Table régionale des services éducatifs de Montréal et le MELS. Ses travaux visent, entre autres, à définir le rôle et les mandats du bibliothécaire scolaire dans une commission scolaire, à élaborer un protocole de partenariat entre le réseau des bibliothèques municipales et scolaires, et à élaborer des documents structurants destinés aux directions d'école pour les soutenir dans leurs démarches d'intégration de la bibliothèque dans la vie pédagogique de l'école. Une autre Table, non officielle, a aussi vu le jour en Montérégie, alors que d'autres régions sont en train de s'organiser.

Pour accroître sa crédibilité et dépoussiérer son image rétrograde très répandue dans le milieu scolaire, le bibliothécaire doit impérativement adopter une posture professionnelle qui implique certaines qualités intrinsèques: une ouverture d'esprit et la capacité à questionner, puis à renouveler ses pratiques. Être professionnel fait référence à une série de gestes et d'attitudes qui supposent une certaine volonté du travail bien fait et une démarche personnelle constante de formation continue. Il ne suffit pas de mettre un diachylon sur le bobo d'un enfant pour être médecin, comme il ne suffit pas de classer des livres pour être bibliothécaire, ou se contenter de bien faire son travail pour être professionnel.

On me permettra de terminer sur une note plus personnelle. Dans mes offres de service aux divers intervenants dans les écoles, une certaine tension se relâche lorsque je me présente comme une bibliothécaire non conventionnelle, ouverte sur la pédagogie et le monde virtuel. L'image négative et cousue de préjugés qu'ont une très grande proportion d'intervenants du milieu scolaire à notre égard doit être radicalement transformée: c'est un combat quotidien et de longue haleine. L'école n'est pas au service de la bibliothéconomie, c'est la bibliothéconomie qui doit être au service de l'école et de ses élèves. L'inversion n'est pas banale et constitue même un enjeu crucial de la survie de notre profession qui met en relief les défis qui sont nôtres. Nous n'aurons pas une troisième chance de nous faire valoir. C'est maintenant qu'il faut questionner notre conformisme, nous libérer de nos œillères, investir de nouvelles avenues et nous exposer volontairement à de nouvelles approches. $\odot$

\section{Sources consultées}

Beaulac, Jacqueline, Paulette Bernhard et Réal Gaudet. 1991. La bibliothèque scolaire, mission et objectifs. Montréal : Association du personnel des services documentaires scolaires (APSDS).

Charest, Diane, et al. 2008. Evvaluation de programme. Plan d'action sur la lecture à l'école. Rapport d'évaluation. Québec : Ministère de l'Éducation, du Loisir et du Sport (MELS).

Klinger, D.A. et al. 2009. Exemplary School Libraries in Ontario. A study by Queen's University and People for Education. Toronto : Ontario Library Association. En ligne à < www.accessola.com/ osla> (consulté le 13 avril 2010).

Manifeste de l'UNESCO de la bibliothèque scolaire. 2000. En ligne à

<archive.ifla.org/VII/s11/pubs/mani-f.htm> (consulté le 21 avril 2010).

Michaud, Pierre, Brigitte Moreau et Louise Paquin. 1997. La bibliothèque de l'école : tâches et compétences. Montréal : Association du personnel des services documentaires scolaires (APSDS).

Moreau, Brigitte. 2010. Définition du poste de bibliothécaire à la Commission scolaire de la Pointe-de-l'île (Titre provisoire). Document de travail interne en cours d'élaboration.

Normes IFLA pour les bibliothèques scolaires. 2004. En ligne à

<archive.ifla.org/VII/s11/pubs/sguideo2-f.pdf> (consulté le 21 avril 2010).

Primary School Library Charter. 2010. Wanborough, UK: School Library Association.

Québec. Ministère de l'Éducation, du Loisir et du Sport. Plan d'action sur la lecture à l'école. 2008. En ligne à <www.mels.gouv. qc.ca/lecture/> (consulté le 25 février 2011).

Rapport de la Commission royale d'enquête sur l'enseignement dans la province de Québec [Rapport Parent]. 1963-1964. 5 tomes. En ligne à <classiques.uqac.ca/contemporains/quebec_commission_parent/commission_parent.html $>$ (consulté le 19 février 2011).

Together for Learning. School Librairies and Emergence of the Learnings Commons. A vision for the 21st Century. 2010. Toronto : Ontario Library Association and Ontario School Library Association. En ligne à <www.accessola.com/data/6/rec_docs/677_ OLATogetherforLearning.pdf> (consulté le 13 avril 2010).

8. Neuf bibliothécaires proviennent de la Commission scolaire de Montréal (CSDM), quatre de la Commission scolaire Marguerite-Bourgeoys (CSMB), un de la Commission scolaire de la Pointe-de-l'̂lle (CSPI), deux de la Commission scolaire English-Montreal (CSEM/ENSB) et deux de la Commission scolaire Lester B. Pearson (CSLBP/LBPSB). 